\title{
Anamnese, exame clínico e exames complementares como testes diagnósticos
}

\author{
Clinical examination and laboratory investigation as diagnostic tests
}

\author{
Isabela M. Benseñor
}

Benseñor IM. Anamnese, exame clínico e exames complementares como testes diagnósticos / Clinical examination and laboratory investigation as diagnostic tests. Rev Med (São Paulo). 2013;00(4):236-41.

RESUMO: As principais ferramentas de que o médico dispõe para fazer o diagnóstico do paciente são anamnese e exame clínico. Hampton et al. em 1970 mostrou, em 80 pacientes ambulatoriais na Inglaterra que a anamnese isolada era responsável por $82,5 \%$ dos diagnósticos, exame clínico por mais $8,75 \%$ e exames complementares por mais $8,75 \%$. Estudo realizado no HCFMUSP mostrou que a anamnese era responsável por $40,4 \%$ dos diagnósticos, exame clínico por mais $29,4 \%$ e exames complementares por mais $29,5 \%$. O exame de laboratório ou de imagem, sempre traz informação sobre a sensibilidade e a especificidade do método; mas não se costuma ensinar a sensibilidade e a especificidade de dados de anamnese ou de manobras do exame clínico. Os resultados de um teste diagnóstico quando comparados a um padrão-ouro permitem a construção de uma table 2 x 2 com quatro possíveis interpretações dos resultados: (1) verdadeiro-positivo, quando positivo na presença da doença; (2) falso-positivo, se o teste revelar-se positivo em paciente sem doença; (3) verdadeiro-negativo, se o teste é negativo e o indivíduo não tem doença; (4) falso-negativo, se o teste é negativo na presença de doença. A partir desses dados pode-se calcular a sensibilidade, a especificidade, o valor preditivo positivo e negativo e as razões de verossimilhança positiva e negativa. A interpretação das informações coletadas na anamnese e no exame clínico como testes diagnósticos refina seu papel na investigação clínica em relação ao diagnóstico final. Os gastos com saúde são finitos e limitados ao orçamento público, e mesmo em um hospital terciário há restrições à realização desses exames causada pelo desbalanço entre demanda e capacidade de realização dos exames. A utilização desse tipo de ferramenta permite priorizar os pacientes que mais precisarão do exame complementar, e como consequência, leva a um melhor gerenciamento do sistema de saúde como um todo.

DESCRITORES: Anamnese; Exames médicos/prevenção \& controle; Exame físico; Testes diagnósticos de rotina.

\begin{abstract}
The main tools that a physician uses for medical diagnosis are clinical history and clinical examination. Hampton et al. in 1970 showed in 80 English outpatients that clinical history was responsible by $82.5 \%$ of medical diagnosis, clinical examination for more $8.75 \%$, and other tests for more $8.75 \%$. Study done in HCFMUSP showed that clinical history was responsible for $40.4 \%$ of medical diagnosis, clinical examination for more $29.4 \%$ e other tests for more $29.5 \%$. A laboratory test or image procedure always brings information about sensitivity and specificity of the method. However, it not common to teach the sensitivity or specificity of information obtained at clinical history or clinical examination. Results from a diagnostic test compared to a gold-standard permit the contruction of a $2 \times 2$ table with four possible interpretations of the results: (1) true positive, when test is positive in the presence of disease; (2) false-positive when it is positive in the absence of disease; (3) true negative when test is negative in the absence of disease; false-negative, when test is negative in the presence of disease. Based on these possibilities it is possible to calculate sensititivity, specificity, positive and negative predictive values, and positive and negative likelihood ratios. Interpretation of the information collected as part of clinical history or examination as diagnostic tests refine their role in the clinical investigation and final diagnosis. Health costs are finite and limited to the public budget, and even in a tertiary care facility there are restrictions to the execution of these tests caused by an imbalance among demand and the capacity to perform the tests. The use of this type of tool permit the use of the tests in patients with the greatest necessity of them, and the main consequence is a better management of the health system as a whole.
\end{abstract}

KEYWORDS: Medical history taking; Medical examination/ prevention \& control; Physical examination; Diagnostic tests, routine.

Professora Associada da Faculdade de Medicina da Universidade de São Paulo.

Endereço para correspondência: Hospital Universitário. Centro de Pesquisa Clínica - $3^{\circ}$ andar. Av. Lineu Prestes, 2565. Cidade Universitária. São Paulo, SP. E-mail: isabensenor@gmail.com 
A principal ferramenta de que o médico dispõe para fazer o diagnóstico das queixas apresentadas pelo paciente são a anamnese e o exame físico. Estudos da década de 70 já mostravam a importância da anamnese e do exame clínico para o diagnóstico. Hampton et al. ${ }^{1}$, no cenário da medicina ambulatorial inglesa, mostrou em 80 pacientes que a anamnese isolada era responsável por $82,5 \%$ dos diagnósticos, anamnese mais exame físico eram responsáveis por mais $8,75 \%$ e que anamnese, exame físico e exames complementares eram responsáveis por mais $8,75 \%$. Sandler ${ }^{2}$, também na Inglaterra, mostrou em 630 pacientes que a anamnese era responsável por $56 \%$ dos diagnósticos, anamnese mais exame físico por mais $17 \%$ e o restante era responsabilidade de exames complementares dirigidos à queixa específica do paciente. Na época, ao ser encaminhado para uma unidade de referência no serviço de saúde inglês, o paciente era submetido a uma série de exames de rotina que incluíam hemograma e urina I, entre outros, sempre com resultados negativos e levando ao aumento dos custos. A conclusão do estudo é que não se deve pedir uma lista fixa de exames e sim, solicitar os exames complementares em função da queixa.

Em 1992, Peterson et al. ${ }^{3}$ fez um estudo semelhante nos Estados Unidos com 80 pacientes concluindo que a anamnese é responsável por $76 \%$ dos diagnósticos, anamnese associada ao exame físico por mais $12 \%$ dos diagnósticos e anamnese, exame físico mais exames complementares por mais $11 \%$ dos casos.

Em 2000, Roshan e Rao ${ }^{4}$, mostraram em estudo realizado na Índia que entre 100 pacientes, a anamnese foi responsável pelo diagnóstico de 78,5\%, anamnese associada ao exame físico por mais $8,2 \%$ dos diagnósticos e anamnese associada aos exames físico e complementares por mais $13,3 \%$ dos diagnósticos. A Tabela 1 mostra esses dados de forma comparativa incluindo os resultados de um estudo brasileiro realizado no Hospital das Clínicas da Universidade de São Paulo5.

Tabela 1. Dados comparativos dos cinco estudos que avaliaram o papel da anmnese, exame clínico e exames complementares no diagnóstico clínico

\begin{tabular}{l|c|c|c|c|c}
\hline & Hampton et al. & Sandler & Petersen et al. & Roshan e Rao & Benseñor et al. \\
\hline Ano & 1975 & 1979 & 1992 & 2000 & 2003 \\
Número de pacientes & $80 \%$ & 630 & 80 & 98 & 95 \\
\hline \multicolumn{5}{l}{ Contribuição (\%) } \\
\hline Anamnese & 82,5 & 56 & 76 & 78,6 & 40,4 \\
Exame físico & 8,75 & 17 & 12 & 8,2 & 29,4 \\
Exames complementares & 8,75 & 23 & 11 & 13,2 & 29,5 \\
Total & 100 & 96 & 99 & 100 & 98,9 \\
\hline
\end{tabular}

Esse estudo mostrou que a anamnese foi responsável pelo diagnóstico de $60 \%$ dos pacientes, anamnese mais exame clínico por mais $25 \%$, e anamnese, exame clínico e exames complementares por mais $15 \%$ dos diagnósticos 5 . Os dados mostram que em ambulatório de pronto atendimento dentro de um hospital de referência, a anamnese e o exame físico ainda são as principais ferramentas de que o médico dispõe.

\section{Avaliação dos dados de anamnese, exame clínico e exames complementares}

De forma geral, quando nos ensinam sobre um exame de laboratório ou de imagem, sempre recebemos a informação sobre a sensibilidade e a especificidade do teste; mas quando pensamos em um dado de anamnese ou de alguma manobra do exame clínico nunca temos essa informação emboratanto os dados da anamese quanto os dados do exame clínico também tenham suas respectivas sensibilidade e especificidade. Isso de uma forma geral não é ensinado no curso médico.
Por que complicar a semiologia com conceitos que vem da epidemiologia para avaliação de testes diagnósticos? Ao invés de complicar, o conhecimento da sensibilidade de uma manobra do exame clínico aumenta capacidade do médico fazer diagnósticos em uma primeira consulta.

\section{Valorização da anamnese e do exame físico}

Na década de 90 foi iniciado um movimento a favor da implementação de uma metodologia científica rigorosa aos estudos diagnósticos com avaliação de todas as informações da anamese e manobras do exame físico na atenção primária, secundária e terciária. Colaborando com essa iniciativa, a revista JAMA (Journal of the Americam Medical Association) iniciou em 1992, uma série de artigos chamada The Rational Clinical Examination que discutiam a sensibilidade e a especificidades de vários dados de anamnese e do exame clínico. No primeiro artigo da série intitulado The science of the art of the clinical examination, David Sackett ${ }^{6}$ que foi o principal criador dessa iniciativa afirma que a anamnese e o exame físico dão 
ao médico todas as ferramentas de que ele necessita para fazer o diagnóstico, permite que sejam afastadas hipóteses diagnósticas, e permite ainda a identificação de pacientes em estágios iniciais de doenças que poderiam levar a morte. Além disso, tirar uma história e realizar um exame físico são elementos venerados da arte da medicina, sendo a melhor série de testes diagnósticos que já existiram. Entretanto, ele também acrescenta que existe uma ciência por trás da arte da medicina começando pela particularização em um paciente específico da nossa experiência prévia com grupos similares de pacientes, utilizando-se dos conhecimentos da literatura. Portanto, atualmente, com o uso da sensibilidade, da especificidade, dos valores preditivo positivo e negativo e das razões de verossimilhança positiva e negativa, que utilizam os conhecimentos de probabilidade condicional descritos pelo reverendo Thomas Bayes há dois séculos atrás, ciência e arte se misturam e se completam? ${ }^{7}$.

Após 5 anos de lançamento da série tinham sido publicados 21 artigos e os autores do editorial The clinical examination - an agenda to make it more rational comentam que apesar do sucesso da série eles viam com grande ironia a falta de investimento na melhora dos conhecimentos sobre o valor da anamnese e do exame físico comparados ao grande investimento que se fez no mesmo período na avaliação de testes diagnósticos, muitos dos quais desnecessários, se fosse gasto algum tempo, estudando-os ${ }^{7}$. Os objetivos dessa série de artigos foram buscar a identificação dos achados de anamnese e exame físico que são úteis ou inúteis baseados na qualidade da evidência clínica, além de estimular novas pesquisas na área. Essa série de artigos do JAMA ainda continua embora com uma frequência de publicação mais baixa sendo publicados dois artigos em 2013, avaliou, a luz das melhores evidências, vários dados de anamnese e manobras do exame físico incluindo uma avaliação baseada em evidências das lombalgias, da esplenomegalia, da palpação do fígado, da pressão venosa central, do melanoma, do infarto do miocárdio, do aneurisma de aorta abdominal, da hipovolemia, da regurgitação aórtica, da meningite aguda, do câncer de mama, do baqueteamento de dedos, da síndrome do túnel do carpo e da lesão de ligamentos do joelho, entre outros temas $^{10-22}$.

Além dessa série do JAMA, outros autores têm publicado artigos avaliando de forma científica a arte da anamnese e do exame físico em várias áreas médicas, mas ainda são poucos os estudos nessa área ${ }^{23-31}$.

\section{A epidemiologia e o diagnóstico}

Para interpretação de um teste diagnóstico é necessário o conhecimento de algumas definições a partir de uma tabela $2 \times 2$. Os resultados de um teste diagnóstico permitem quatro possíveis interpretações: (1) verdadeiro-positivo, quando positivo na presença da doença; (2) falso-positivo, se o teste revelar-se positivo em paciente sem a doença; (3) verdadeironegativo, ao excluir possibilidade da doença em indivíduo que realmente não a possui; (4) falso-negativo, ao descartar a doença ela esta presente (Tabela 2$)^{32}$.

Tabela 2. Interpretações possíveis para o resultado de um teste diagnóstico

\begin{tabular}{lll}
\hline & Presente & Ausente \\
\hline \multirow{2}{*}{ Positivo } & Verdadeiro- positivo & Falso-positivo \\
& 13 (a) & 1 (b) \\
\multirow{2}{*}{ Negativo } & Falso-negativo & Verdadeiro- negativo 17 \\
& 5 (c) & (d) \\
\hline
\end{tabular}

Usando como base os dados de artigo publicado que avaliou a acurácia do auto-relato de DPOC pelo paciente, do antecedente de tabagismo com quantificação em maços/ano quando a resposta era positiva, presença de sibilos (auscultados durante a expiração em quatro áreas pré-determinadas nas bases e ápices do pulmão e da tempo expiratório forçado avaliado da seguinte forma: pede-se para o paciente fazer uma inspiração máxima e depois expirar o ar pela boca de maneira forçada ${ }^{33}$. O médico com o estetoscópio sobre a traqueia contava o tempo da expiração audível. Cada uma dessas medidas foi considerada como um teste diagnóstico sendo comparado com o padrão-ouro representado pela espirometria. Para cada teste calculou-se a sensibilidade (sensibilidade $=\mathrm{a} / \mathrm{a}+\mathrm{c}$ ), $\mathrm{a}$ especificidade (especificidade $=\mathrm{d} / \mathrm{c}+\mathrm{d}$ ), o valor preditivo positivo $(\mathrm{VPP}=\mathrm{a} / \mathrm{a}+\mathrm{b})$ e o valor preditivo negativo (VPN $=\mathrm{d} / \mathrm{c}+\mathrm{d})$. Além desses valores também foram calculadas a razão de verossimilhança positiva $(\mathrm{RVP}=$ sensibilidade/1especificidade) e a razão de verossimilhança negativa (RVN =1-sensibilidade/especificidade). A RVP é um número que a partir da probabilidade pré-teste calcula a probabilidade pós-teste do paciente ter a doença. A probabilidade pré-teste nada mais é que a prevalência da doença em indivíduos de mesmo sexo e faixa etária e pode ser obtida a partir de dados da literatura. Quando o resultado do teste é positivo (seja ele um dado de anamnese, uma manobra do exame clínico ou um exame laboratorial ou complementar) calculase a RVP. Usando-se o nomograma de Fagan a partir da probabilidade pré-teste e da RVP acha-se a probabilidade pós-teste. Embora esse processo pareça complicado artigos como o dessa série do JAMA já trazem os valores da RVP calculados para várias manobras do exame clínico ou para vários dados da anamnese. Por exemplo, na Tabela 3 estão os valores da RVP para vários dados de história e do exame clínico que são importantes no diagnóstico das lombalgias. Por exemplo, para um paciente que chega com queixa de dor lombar importante e na anamnese descobre-se que ele usa cortiscosteróides há muitos anos, a RVP calculada a partir de estudos prévios é de 12 (Tabela 3). Quando você tem uma RVP acima de 10, independente da probabilidade pré-teste de fratura vertebral nesse paciente, com esse valor de RVP a probabilidade pós-teste determinada pelo nomograma de Fagan $^{34}$ (Figura 1) será próxima de 100\% e a melhor conduta nesses casos é concluir que esse paciente 
Benseñor IM. Anamnese, exame clínico e exames complementares como testes diagnósticos.

tem uma fratura vertebral e já trata-lo de forma dequada. Quanto mais alta a RVP melhor, a probabilidade pós-teste fica próxima de $100 \%$ e é possível fechar o diagnóstico do paciente antes que outros exames sejam realizados (Tabela 4). Quando o resultado do teste é negativo, calcula-se a razão de verossimilhança negativa (RVN). Em relação a
RVN, quanto mais próxima de zero melhor. Quando a RVN é próxima de zero, independente do valor da probabilidade pré-teste, o valor da probabilidade pós-teste será próximo de zero e é possível afastar o diagnóstico de uma doença determinada naquele paciente (Tabela 4).

Tabela 3. Sensibilidade, especificidade, razão de verossimilhança positiva e negativo de dados da anamnese e de manobras do exame clínico importantes para o diagnóstico das doenças que causam lombalgia*

\begin{tabular}{|c|c|c|c|c|c|}
\hline Doença & Dado da anamnese & Sensibilidade & Especificidade & RVP & RVN \\
\hline \multirow{4}{*}{ Câncer } & Idade $>50$ anos & 0,77 & 0,71 & 2,7 & 0,32 \\
\hline & História prévia de câncer & 0,31 & 0,98 & 14,7 & 0,70 \\
\hline & Perda de perda inexplicável & 0,15 & 0,94 & 2,7 & 0,90 \\
\hline & Ausência de melhora após 1 mês de tratamento & 0,31 & 0,90 & 3,0 & 0,77 \\
\hline \multirow{2}{*}{ Fratura } & Idade $\geq 70$ anos & 0,22 & 0,96 & 5,5 & 0,81 \\
\hline & Uso de corticosteróide & 0,06 & 0,995 & 12,0 & 0,94 \\
\hline Herniação de disco & Presença de lombociatalgia & 0,95 & 0,88 & 7,9 & 0,06 \\
\hline Espondilite anquilosante & Expansão do tórax a inspiração $\leq 2,5 \mathrm{~cm}$ & 0,09 & 0,99 & 9,0 & 0,92 \\
\hline \multirow{2}{*}{ Estenose de medula } & Alargamento da base ao andar & 0,43 & 0,97 & 14,3 & 0,59 \\
\hline & Ausência de dor na posição sentada & 0,46 & 0,93 & 6,6 & 0,58 \\
\hline
\end{tabular}

Adaptado da referência 10; RVP = razão de verossimilhança positiva; RVN = razão de verossimilhança negativa.

Tabela 4. Significado das razões de probabilidade positiva e negativa

Razão de probabilidades maior do que 10 ou menor do que 0,1: introduz mudanças conclusivas da probabilidade pré-teste para a pósteste.

Razão de probabilidades de 5 a 10 e 0,1 a 0,2: determina mudanças moderadas da probabilidade pré-teste para a pós-teste.

Razão de probabilidades de 2 a 5 e 0,5 a 0,2: gera mudanças pequenas, mas ainda assim importantes, na probabilidade pós-teste.

Razão de probabilidades de 1 a 2 e 0,5 a 1: altera pouco a probabilidade pós-teste e de forma raramente importante.

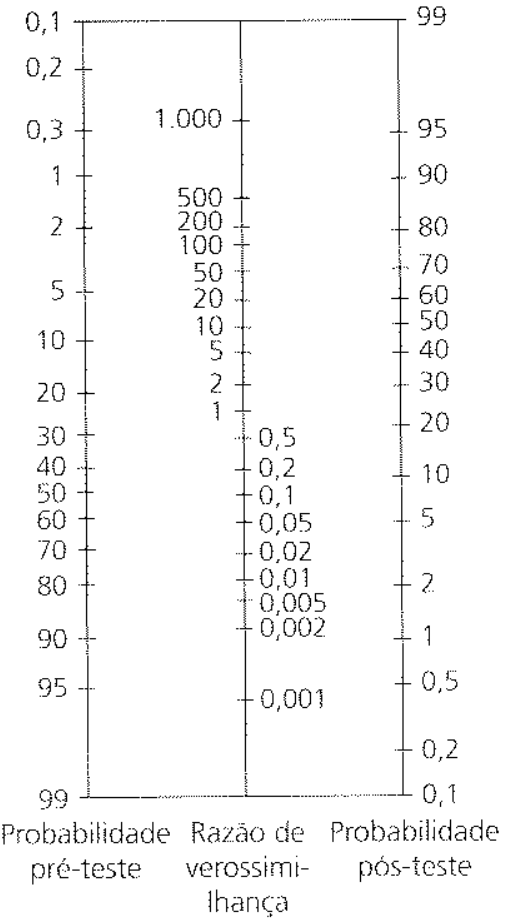

Figura 1. Nomograma de Fagan

\section{Como usar o nomograma de Fagan?}

Deve-se ancorar uma régua na margem esquerda do nomograma, no caso, no ponto onde está o valor da probabilidade pré-teste da doença, rodar a régua até que cruze com a linha central do nomograma na razão de probabilidade positiva ou negativa que já foi calculada ou que foi encontrada em dados da literatura. O ponto de intersecção da régua com a terceira linha, presente à esquerda, identifica o valor que corresponde à probabilidade pós-teste. Utiliza-se o mesmo procedimento para a RVN.

Voltando ao caso do artigo sobre DPOC. De todos os fatores estudados o auto-relato de DPOC, a ausculta de sibilos pelo médico e um tempo expiratório forçado $(T E F) \geq 9$ se associaram a RVP de 4,4, 2,9 e 4,6 e a RVN (quando a resposta para tabagismo foi negativa, não se auscultaram sibilos e o tempo expiratório forçado foi < 9) foi de $0.5,0.8$ e 0,8 , respectivamente. Nenhuma das RVP ficou acima de 10, mas se forem combinados os resultados, o paciente que tem história positiva de DPOC, tinha sibilos na ausculta e um $\mathrm{TEF} \geq 9$ apresentava uma RVP de 59 de realmente ter DPOC e a RVN de 0,3 de não apresentar o diagnóstico. Isso permitirá o fechamento do diagnóstico antes da espirometria. Isso não quer dizer que 
não devemos fazer a espirometria. Em algumas situações em que a disponibilidade do exame complementar é difícil ou vai demorar muito para ser realizado, isso ajuda na decisão pela melhor conduta no primeiro atendimento ao paciente. Isso também permitirá a priorização dos exames complementares para os pacientes que tem mais necessidade.

\section{Anamnese e exame clínico versus exames complementares}

Não existe nenhum conflito entre a anamese, o exame clínico e os exames complementares. Todos tem seu papel na investigação diagnóstica. Mas há sim uma ordenação que deve ser mantida e começar pela anamese e terminar pelos exames complementares. A interpretação das informações coletadas na anamnese e no exame clínico como testes diagnósticos refina o papel desses componentes

\section{REFERÊNCIAS}

1. Hampton JR, Harrison MJG, Mitchell JRA, Prichard JS, Seymor C. Relative contributions of history-taking, physical examination, and laboratory investigation to diagnosis and management of medical outpatients. BMJ. 1975;2:486-9. Available from: http://www.ncbi.nlm.nih.gov/pmc/articles/ PMC1673456/pdf/brmedj01449-0038.pdf

2. Sandler G. The importance of the history in the medical clinic and the cost of unnecessary tests. Am Heart J. 1980;100:92831.

3. Peterson M, Holbrook JH, De Von Hales, Smith NL, Staker LV. Contributions of the history, physical examination, and laboratory investigation in making medical diagnoses. West J Med. 1992;156:163-5. Available from: http://www.ncbi. nlm.nih.gov/pmc/articles/PMC1003190/pdf/westjmed000900053.pdf

4. Roshan R, Rao AP. A study of relative contributions of the history, physical examination and investigations in making medical diagnosis. JAPI. 2000;48:771-5.

5. Bensenor IM. O papel da anamnese, do exame clinico e dos exames complementares no diagnóstico clínico [tese]. São Paulo: Faculdade de Medicina, Universidade de São Paulo; 2003.

6. Sackett DL. The science of the art of the clinical examination. JAMA. 1992;267(19):2650-2. doi:10.1001/ jama.1992.03480190092040.

7. Simel DL, Rennie D. The clinical examination - an agenda to make it more rational. JAMA. 1997;277:572-574-8. doi:10.1001/jama.1997.03540310070037.

8. Hollands H, Johnson D, Hollands S, Simel DL, Jinapriya D, Sharma S. Do findings on routine examination identify patients at risk for primary open-angle glaucoma? The da investigação clínica em relação ao diagnóstico final. Saber que a percussão do baço tende a ser positiva (teste falso-positivo) no paciente que acabou de se alimentar por causa do estômago cheio e não porque o baço está realmente aumentado, auxilia na investigação diagnóstica. Antes de pedir um exame de ultrassonografia para confirmar o diagnóstico de esplenomegalia, talvez seja melhor fazer a percussão no dia seguinte com o paciente em jejum. Outra questão é a da priorização dos exames complementares para quem tem mais necessidade. Os gastos com saúde são finitos e limitados ao orçamento de prefeituras, estados e do país como um todo. Mesmo em um hospital terciário há restrições a realização desses exames causada pela grande procura e o número de aparelhos e de operadores disponíveis. A utilização desse tipo de ferramenta permite uma priorização dos pacientes que mais precisarão do exame complementar, e como consequência, leva a um melhor gerenciamento do sistema de saúde como um todo. rational clinical examination systematic review. JAMA. 2013;309(19):2035-42. doi: 10.1001/jama.2013.5099.

9. Crochet JR, Bastian LA, Chireau MV. Does this woman have an ectopic pregnancy the rational clinical examination systematic review. JAMA. 2013;309(16):1722-9. doi: 10.1001/jama.2013.3914.

10. Deyo RA, RainvilleJ, Kent DL. The rational clinical examination. What can the history and physical examination tell us about low back pain? JAMA. 1992;208:760-6. doi:10.1001/jama.1992.03490060092030.

11. Grover AS, Barkun NA, Sackett DL. The rational clinical examination. Does this patient have splenomegaly? JAMA. 1993;270:2218-45. doi:10.1001/ jama.1993.03510180088040.

18. Naylor CD. The rational clinical examination.Physical examination of the liver. JAMA. 1994;271:1859-65. doi:10.1001/jama.1994.03510470063036.

12. Cook D, Simel, DL. The rational clinical examination. Does this patient have abnormal venous pressure? JAMA. 1996;28:630-4. doi:10.1001/jama.1996.03530320054034.

13. Whited JD, Grichnik JM. The rational clinical examination. Does this patient have a mole or a melanoma? JAMA. 1998;279:696-701. doi:10.1001/jama.279.9.696.

14. Akbar AP, Hemmelgarn BR, Guyatt GH, Simel DL. Is this patient having a myocardial infarction? JAMA. 1998;280:1526-63. doi:10.1001/jama.280.14.1256.

15. Lederle FA, Simel DL. The rational clinical examination.Does this patient have aortic aneurysm? JAMA. 1999;281:77-82. doi:10.1001/jama.281.1.77.

16. McGee S, Abernethy WB. Simel DL. The rational clinical examination. Is this patient hypovolemic? JAMA. 
Benseñor IM. Anamnese, exame clínico e exames complementares como testes diagnósticos.

1999;281:1022-9. doi:10.1001/jama.281.11.1022.

17. Choudhry NK, Etchells EE. The rational clinical examination. Does this patient have aortic regurgitation? JAMA. 1999;281:2231-8.

18. Attia J, Hatala R, Cook DJ. Wong JG. The rational clinical examination. Does this adult patient have acute meningitis? JAMA. 1999;282:175-81. doi:10.1001/jama.282.2.175

19. Barton MB, Harris R, Fletcher SW. The rational clinical examination. Does this patient have breast cancer? The screening clinical breast examination: Should it be done? How? JAMA. 1999;282:1270-80. doi:10.1001/ jama.282.13.1270.

20. D'Arcy CA, McGee S. The rational clinical examination. Does this patient have carpal tunnel syndrome? JAMA. 2000;283:3110-7. doi:10.1001/jama.283.23.3110.

21. Myers KA, Farquhar DRE. The rational clinical examination. Does this patient have clubbing? JAMA. 2001;286:341-7. doi:10.1001/jama.286.3.341.

22. Solomon DH, Simel DL, Mates DW, Katz JN, Schaffer JL. The rational clinical examination. Does this patient have a torn meniscus or ligament of the knee. Value of the physical examination. JAMA. 2001;286:1610-20. doi:10.1001/ jama.286.13.1610.

23. Buzzell KA, Boller MW. Relation of medical history and physical examination to the diagnosis of foot disorders. J Am Osteopath Assoc. 1969;69:388-92.

24. Criqui MH, Fronek A, Melville RK, Barrett-Connor E, Gabriel $\mathrm{S}$. Thsensitivity, specificity, and predictive value of traditional clinical evaluation of peripheral arterial disease: results form noninvasive testing in a defined population. Circulation. 1985;71:516-22. doi: 10.1161/01.CIR.71.3.516

25. Sox HC, Hickam DH, Marton KI, Moses LN, Skeff KM, Sox MS. Using the patient's history to estimate the probability of coronary artery disease: a comparison of primary care and referral practices. Am J Med. 1990; 89:7-14. http://dx.doi. org/10.1016/0002-9343(90)90090-Z
26. Heckerling PS, Wiener SL, Wolfkiel CJ, Kushner MS, Dodin EM, Jelnin V, Fusman B, Chomka EV. Accuracy and reproducibility of precordial precussion and palpation for detecting increased left ventricular end-diastolic volume and mass - a comparison of physical findings and ultrafast computed tomography of the heart. JAMA. 1993;270:1943-8. doi:10.1001/jama.1993.03510160061030.

27. Smith BW, Green GA. Acute knee injuries: Part I. History and physical examination. Am Fam Physician. 1995;51(3):615-21. Available from: http://goo.gl/FZMyD2

28. Cuomo F. The value of the history and physical for shoulder pain [editorial]. West J Med. 1995;163:389-90. Available from: http://www.ncbi.nlm.nih.gov/pmc/articles/PMC1303147/pdf/ westjmed00361-0073.pdf

29. Davis-Joseph B, Tiefer L, Melman A. Accuracy of the initial history and physical examination to stablish the etiology of erectile dysfunction. Urology. 1995;45:498-502. http://dx.doi. org/10.1016/S0090-4295(99)80022-3

30. Cleland JGF, Habib F. Assessment and diagnosis of heart failure. J Intern Med. 1996;239:317-25. doi: 10.1046/j.13652796.1996.462801000.x

31. Drager LF, Abe J, Martins MA, Lotufo PA, Benseñor IM. Impact of clinical experience on quantification of clinical signs at physical examination. J Intern Med. 2003;253:1-7. doi: 10.1046/j.1365-2796.2003.01183.x

32. Hatanaka VMA, Bensenor IM. Avaliação de testes diagnósticos. In: Benseñor IM, Lotufo PA. Epidemiologia aboradagem prática. 2a ed.: São Paulo: Sarvier; 2011. p.272302.

33. Straus S, McAlister FA, Sackett DL, Jonathan JD, on behalf of the CARE-COAD2 Group. Accuracy of history, wheezing, and forced expiratory time in the diagnosis of chronic obstructive pulmonary disease. J Gen Intern Med. 2002;17:684-8. doi: 10.1046/j.1525-1497.2002.20102.x

34. Fagan TJ. Nomogram for Bayes theorem [letter]. N Engl J Med. 1975;293(5):257. doi: 10.1056/NEJM197507312930513 